NASA Technical Memorandum 105843

123625
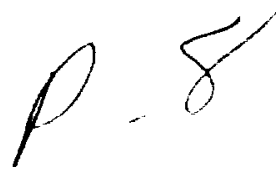

\title{
Description of the SSF PMAD DC Testbed Control System Data Acquisition Function
}

Anastacio N. Baez, Michael Mackin, and Theodore Wright

Lewis Research Center

Cleveland, Ohio

August 1992

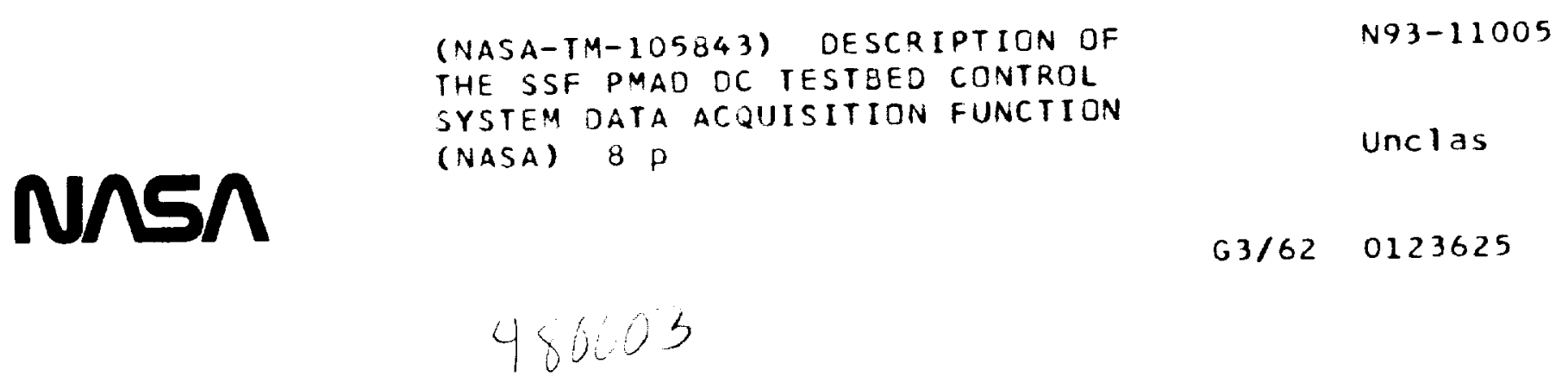





\section{Description of The SSF PMAD DC Testbed Control System Data Acquisition Function \\ Anastacio N. Baez, Michael Mackin, and Theodore Wright \\ National Aeronautics and Space Administration \\ Lewis Research Center \\ Cleveland Ohio 44135}

\begin{abstract}
The NASA Lewis Research Center in Cleveland, Ohio has completed the development and integration of a Power Management and Distribution (PMAD) DC Testbed. This testbed is a reduced scale representation of the end to end, sources to loads, Space Station Freedom Electrical Power System (SSF EPS). This unique facility is being used to demonstrate DC power generation and distribution, power management and control, and system operation techniques considered to be prime candidates for the Space Station Freedom. A key capability of the testbed is its ability to be configured to address system level issues in support of critical SSF program design milestones. Electrical power system control and operation issues like source control, source regulation, system fault protection, end-to-end system stability, health monitoring, resource allocation and resource management are being evaluated in the testbed.
\end{abstract}

The SSF EPS control functional allocation between on-board computers and ground based systems is evolving. Initially, ground based systems will perform the bulk of power system control and operation. The EPS control system is required to continuously monitor and determine the current state of the power system. The DC Testbed Control System consists of standard controllers arranged in a hierarchical and distributed architecture. These controllers provide all the monitoring and control functions for the DC Testbed Electrical Power System. Higher level controllers include the Power Management Controller, Load Management Controller, Operator Interface System, and a network of computer systems that perform some of the SSF Ground Based Control Center Operation. The lower level controllers include Main Bus Switch Controllers and Photovoltaic Controllers.
Power system status information is periodically provided to the higher level controllers to perform system control and operation. The data acquisition function of the control system is distributed among the various levels of the hierarchy. Data requirements are dictated by the control system algorithms being implemented at each level. This paper presents a functional description of the various levels of the testbed control system architecture and describes the data acquisition function and the status of its implementation.

\section{Introduction}

The NASA LERC PMAD DC Testbed is a reduced scale representation of the power management and distribution system for the Space Station Freedom The SSF PMAD system consists of DC power generation, DC distribution, and control equipment In its final configuration the testbed will consists of two power channels utilizing of breadboard type components. Power sources and electrical load simulators have been developed to assist with the integration of the various testbed elements and to address system level issues. Power system control and operation issues like source control, source regulation, system fault protection, end-to-end system stability, health monitoring, resource allocation and resource management are being evaluated in the testbed. The testbed main components are: Solar Arrays (SA) and Solar Array Electronic Simulators (SAES), Sequential Shunt Units (SSU), DC Switching Units (DCSU), Main Bus Switching Units (MBSU), DC to DC Converter Units (DDCU), secondary and tertiary switchgear, load converter units, load simulators, and a hierarchical distributed control system. A complete description of the PMAD DC Testbed and its evolution is found in reference [1]. 


\section{Control System Description}

Figure 1 is a block diagram of the PMAD DC Testbed highlighting the control system architecture. The DC Testbed control system is a hierarchical and distributed array of Intel 386 based standard controllers. Each controller is configured with the appropriate Ada software to perform a power control function. The monitoring and control functions, implemented in each node, ensure safe, efficient, and autonomous system operation. The ADA programming language has been selected by the SSF program to provide a flexible platform for efficient control algorithm development and implementation. testbed operators and provides some of the functions that the Data Management System (DMS) will provide in the SSF. Among these are: testbed software and hardware configuration and initialization, power system monitoring, graphical data display, and command and control of all power system components. Currently, one computer performs these functions for both the primary distribution system and the secondary/tertiary systems. In its final configuration the testbed will have two OIS computers like shown in Figure 1. The GBC are a cluster of controllers that will emulate the functionality of the SSF Control Center and the Engineering Support Center (ESC). Their major function is to provide for long term EPS

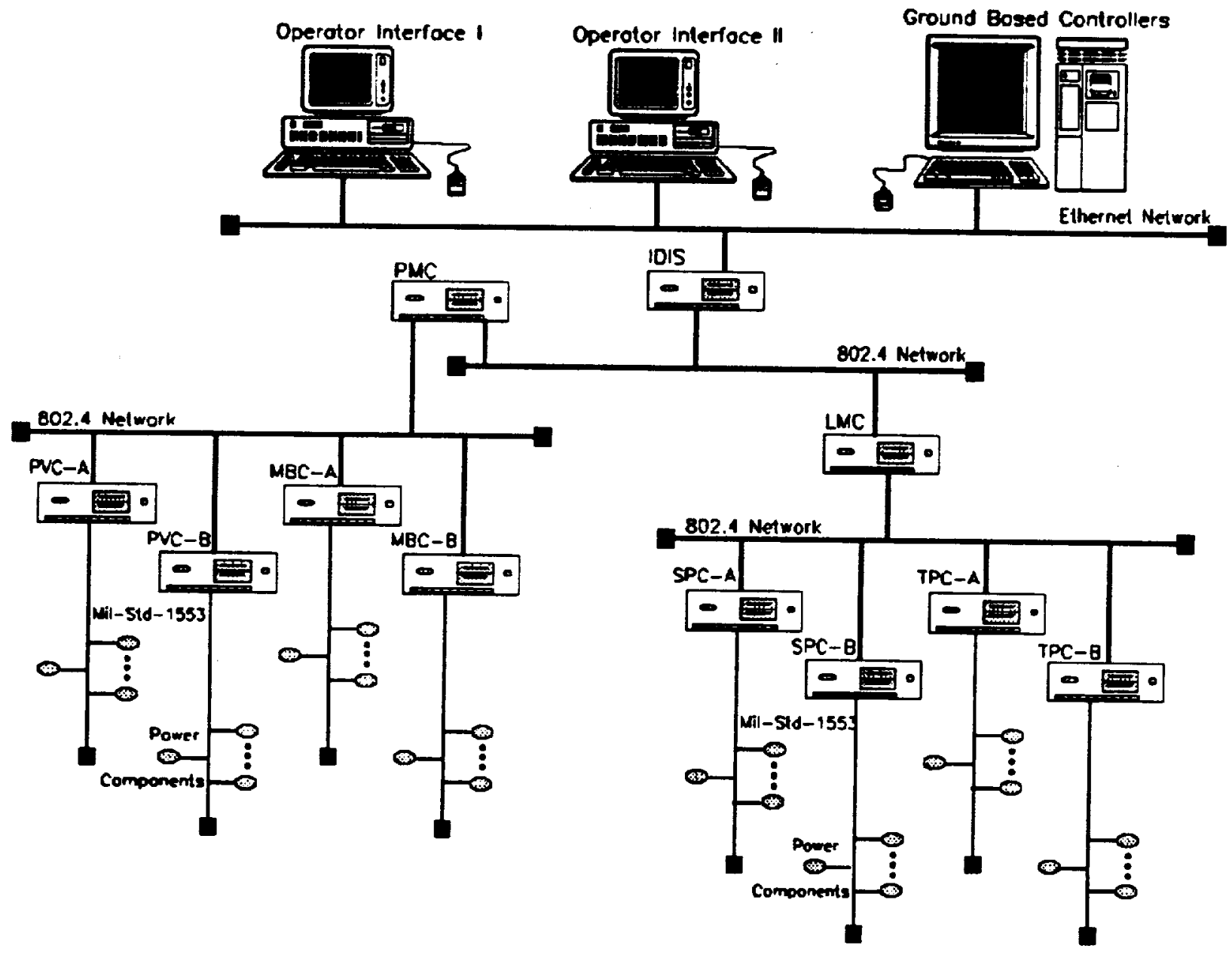

FIGURE 1 PMAD DC Testbed Control System Diagram

At the top of the hierarchical control scheme is the Operator Interface System (OIS) and the Ground Based Controllers (GBC), which are referred to as the Level I controllers. The OIS is the main interface for planning, resource allocation, trend analysis, health monitoring, scheduling, and other power system operation functions. The Level I controllers 
communicate with the Level II controllers via an Integrated Data Interface System (IDIS).

The IDIS provides a standard software and hardware interface between the GBC/OIS function and the Level II controllers. Testbed power system data is accessible to all Level I controllers via the IDIS. High level command and control of the testbed components is performed throughout the IDIS.
Switching Unit (DCSU), and the Sequential Shunt Unit (SSU). The Level III controllers communicate with the power components using a MIL STD 1553B data bus.

The Secondary Distribution Controllers (SDC) and the Tertiary Power Controller (TPC) are also Level III controllers. The SDC and TPC monitor and control the secondary and tertiary distribution systems, respectively. The design and development of the

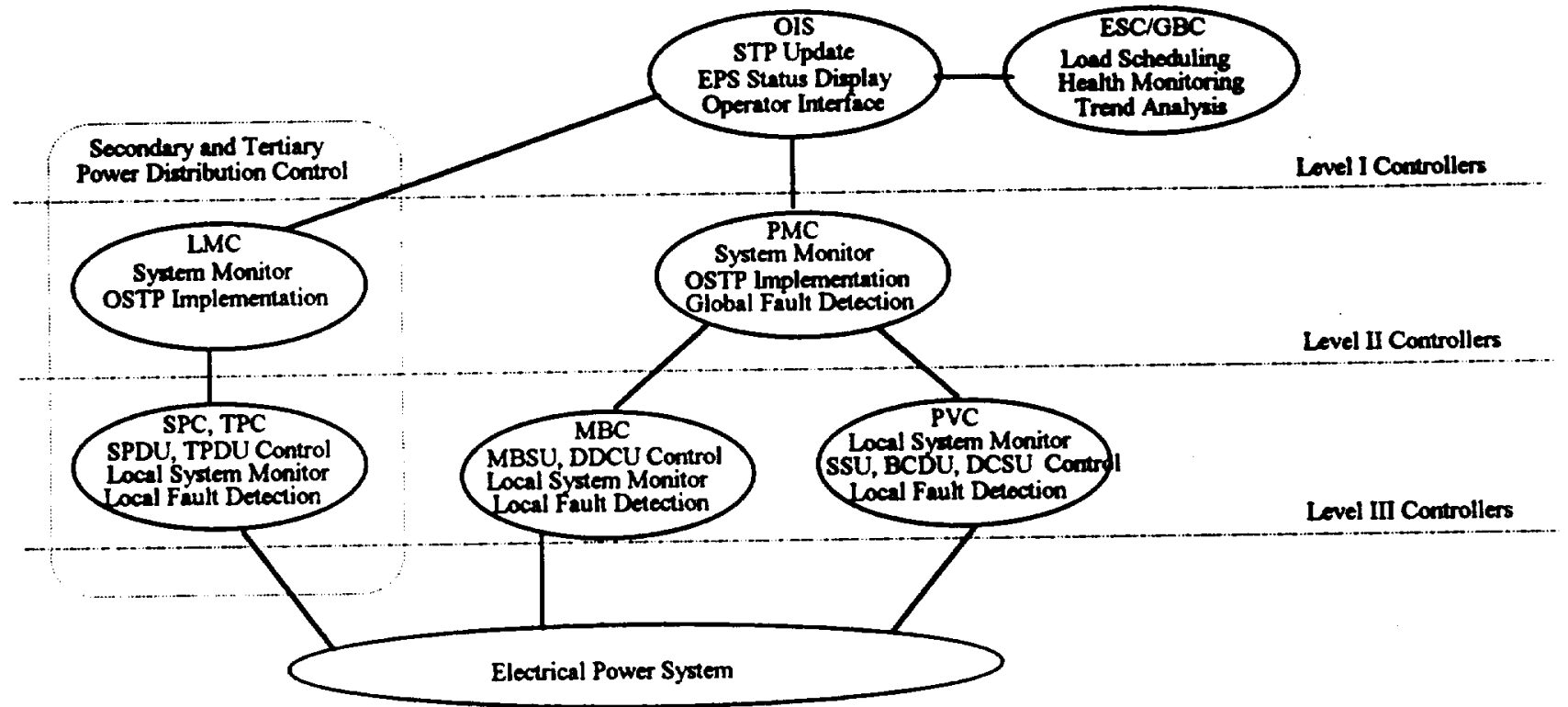

Figure 2 Control System Hierarchical Functional Breakdown

The Power Management Controller (PMC) and the Load Management Controller (LMC) are Level II controllers. Their major functions are to coordinate the low level (level III) controllers and track overall system performance in both, the primary and secondary/tertiary distribution systems. The PMC verifies and implements the On-board Short Term Plan (OSTP), initializes the primary distribution system, performs global system monitoring, and provides power system information to the OIS and GBC. The Level II controllers communicate with the Level III controllers via an 802.4 token bus network.

The Level III controllers, also known as the low level controllers, are the Main Bus Controller (MBC) and the Photovoltaic Controller (PVC). The MBC monitors and controls the Main Bus Suitching Unit (MBSU) and the DC-to-DC Converter Units (DDCUs); the PVC monitors and controls the Battery Charge/Discharge Units (BCDUs), the Direct Current functions performed by these controllers, as well as the LMC, falls within the SSF modules and payloads efforts, which are not the responsibility of the NASA LeRC. However, minimal monitoring and control functions are implemented to ensure safe operation of the power hardware.

\section{Control System Data Requirements}

The control system's two major functions are to continuously monitor the state of the power system and to control the various elements of the testbed. The control system monitoring function is implemented by periodically sampling the power system components' data, smoothing it, and analyzing it for acceptable system performance. The monitoring functional requirements are to ensure safe operation of the power system, to track energy consumption and storage, to detect and verify faults and interruptions, and to 
provide the OIS and GBC with a confident EPS operating state.

The system monitor is distributed among the various levels of the control architecture. Functions that require time critical data are performed as close as possible to the power system element being monitored. Therefore, time critical functions are implemented at the lowest level, level III, of the control architecture. Functions that require global system data and that can tolerate slower response are implemented at the higher levels. The data requirements of each level of the hierarchy are determined by the functions implemented at each level. References [2] and [3] explain in detail the testbed control system design and its functionality. Figure 2 is a diagram of the testbed control system showing the major functions implemented at each level of the architecture.

Data requirements at the Level III are dictated by the fault detection algorithms being implemented. These algorithms require digitally filtered data to smooth out load modulations. These digital filters are implemented using a 100 milliseconds update time. Voltages and currents are filtered at this level. Power components status information (on/off/tripped, temperature) is monitored at the same rate to ensure power availability to the users and to provide for caution and warning messages.

\section{Data Acquisition Function Description}

Data is acquired from the power system component sensors once every 100 milliseconds. The data is then passed through a $2 \mathrm{~Hz}$ digital filter and analyzed for power interruption or overcurrent conditions. The processed data is passed from the lower level controllers to the Power Management Controller or Load Management Controller over the 802.4 token passing communications bus. The PMC and LMC use the data to analyze the power system's performance, verify low level fault detection, and to report on its status to the testbed operators.

LOW LEVEL DATA ACQUISITION - The Level III controllers, which are physically connected to the power components via the 1553B data busses, perform the low level data acquisition.

Each Level III computer uses an electronic timer card to initiated the data acquisition cycle. The cards generate an interrupt every 100 milliseconds. The interrupt triggers a computer process, known as the system monitor, to obtain a block of binary formatted data from all the power components attached to the 1553B data bus. The data is then scaled and passed through a digital low-pass filter for smoothing.
Currently, a $2 \mathrm{~Hz}$ third-order Butterworth filter is used. The system monitor process analyzes the filtered data for abnormal conditions caused by power interruptions, under voltage conditions, bus faults, or line faults. The system monitor can detect "hard" faults caused by devices that have tripped due to overcurrent as well as "soft" faults caused by current values that are outside the expected range.

Data collected by the system monitoring process is packaged together into a data snapshot as part of the control system's global data acquisition capability.

GLOBAL DATA ACQUISITION - Global data acquisition allows the Power Management Controller and Load Management Controller to use the data snapshots obtained from each of the low level controllers. The snapshots stored within the low level controllers are transmitted to the Power Management Controller or Load Management Controller once every second via the 802.4 token passing bus. In the present control software version, the snapshots are time stamped when they are received at this level. Time stamping of testbed data at the level III controllers is being investigated. The data contained within the snapshots is stored in a database that is available for use by high level control processes residing on the Power Management and Load Management controllers, as well as computers connected to the IDIS. Because this database contains data from each of the low level controllers, it is known as the Global Database.

GROUND BASED DATA ACQUISITION Computers outside of the testbed can make use of the data stored in the global database. These computers include the two computers used for operation of the testbed (the Operator Interface System, or OIS), and other computers representing ground based monitoring of the space station. The Ground Based Computers, GBC are part of the Engineering Support Center, ESC, and are called the GBC/ESC computers. This section describes the flow of data from the Global databases on the PMC and LMC to the OIS and GBC/ESC computers.

GRAPHICS ALGORITHMS - A set of Ada tasks running on the PMC (and LMC) are responsible for retrieving the data from the global databases and sending it to the ground based computers. These tasks are referred to collectively as the Graphics Algorithm, because their original purpose was to send data to the OIS to be displayed graphically. There is a separate Graphics Algorithm on the PMC and the LMC. 
The Graphics Algorithm requests the data needed by the OIS and GBC/ESC computers from the global database. This request causes the data to be periodically updated in the global database (if the data was not already being updated as part of a snapshot). The Graphics Algorithm then polls the global database (currently about once per second) to find data that has changed since the last poll. If the data has changed, it is extracted from the database (along with the data's time stamp) and formatted for transfer to the Integrated Data Interface System (IDIS computer)

DATA TRANSFER TO THE IDIS - The IDIS acts as a sort of data crossroads, handling data requests from various OIS and GBC/ESC computers, and making sure that when the data arrives from the PMC and LMC, the appropriate data is directed to the computers that requested it.

The PMC and LMC send the changed data to the IDIS over an additional 802.4 network dedicated to this purpose. Because the OIS and GBC/ESC computers communicate over a TCP/IP Ethernet interface, the IDIS must covert data between these two types of networks. This IDIS software is written in the $\mathrm{CH}$ language, so that commercial network drivers could be used.

DATA TRANSFER FROM IDIS - The IDIS keeps a list of all the data items that can be requested from the testbed. When an OIS or GBC/ESC computer requests a data item, the list is marked so that when new data arrives, the IDIS can send the data only to the computers that requested it. The data is sent via a TCP/IP Ethernet network to the proper computers.

The IDIS merges data requests from the OIS and GBC/ESC computers, so that the graphics algorithm is only notified that a datum is needed once, no matter how many requesters there are. The IDIS also keeps a database holding all the information needed to requests a specific piece of data (if it is not in the snapshot). This information is part of the requests sent to the graphics algorithm. Since this information is appended by the IDIS, the OIS and GBC/ESC computers can requests data by name without having to supply the location of the data (PMC or LMC) and the message need to retrieve the data.

\section{Development Status}

Development of the low level data acquisition scheme has been completed and incorporated into the testbed Ada control software. Reference [4] contains a detail description of the implementation of these functions. In order to have a working system in the testbed at all times, and to ease software development, the IDIS and the global database will be implemented in an evolutionary manner. Each stage of the process is called a prototype. Several prototypes have been identified and tested. Figure 3 shows the latest prototype that is currently operational in the testbed. In this prototype the global database has been integrated into the PMC software and the IDIS basic functions for messages and data routing to the OIS and GBC/ESC computers have been incorporated. Verification of data requirements compliance at the various levels of the control architecture is underway. The Engineering Support Center computer network has been hooked-up to the testbed and various data transfer exercises demonstrated. Final integration and check-out of the data acquisition functions and verification of all the functional requirements is expected to be completed by the end of August of 1992.

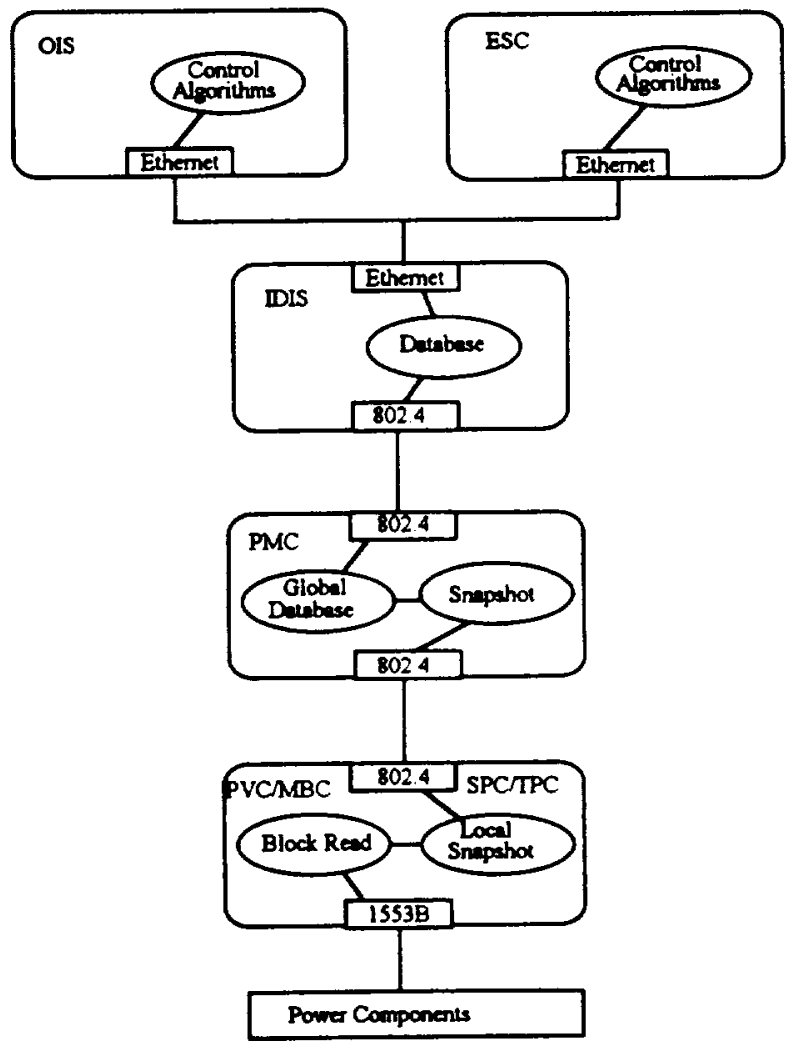

Figure 3 Testbed Data Acquisition Function Prototype 


\section{Conclusions}

Data requirements for the testbed control system are dictated by the functionality being implemented at each level of the architecture. The testbed control system hierarchy data acquisition function is distributed among its various levels. Fast acting control functions, that require time critical data are implemented at the lowest level. These functions require periodic data sampling to assure safe system operation and performance. Slower response control functions are implemented at the higher levels and require data to be reported at least every second. The data acquisition function presented in this paper meets the requirements for a hierarchical and distributed power management and control system. Final integration and check-out of all the capabilities presented in this paper are currently underway in the NASA LeRC PMAD DC Testbed.
References

[1] J. F. Soeder and R. J. Frye, "Overview and Evolution of the LeRC PMAD DC Test Bed", IECEC92 Proceedings, August, 1992.

[2] A. N. Baez and G. L. Kimnach, "Description of the Control System Design for the Space Station Freedom PMAD DC Testbed", IECEC-91 Proceedings, August, 1991.

[3] G. L. Kimnach and A. N. Baez, "Power System Monitoring and Source Control of the Space Station Freedom DC Power System Testbed", IECEC-92 Proceedings, August, 1992.

[4] K. Ludwig, T. Wright, and M. Mackin, "Description of Real-Time Ada Software Implementation of a Power System Monitor For The Space Station Freedom PMAD DC Testbed", IECEC91 Proceedings, August, 1991. 
Public reponting burden for this collection of information is estimated to average 1 hour per response, including the tume lor teviewing instructions. searching existing bata sources, gathering and mainkaining the data needed, and completing and reviewing the colloction of intormatoon. Send comments regarding this burden estimate or any other aspect of this Davis Highway. Suite 1204. Arlington, VA 22202-4302, and to the Oftice of Management and Budget. Paperwork Reduction'Project (0704-0188). Washington, DC 20503.

\begin{tabular}{|l|l|l|}
\hline 1. AGENCY USE ONLY (Leave blank) & $\begin{array}{r}\text { 2. REPOAT DATE } \\
\text { August } 1992\end{array}$ & $\begin{array}{r}\text { 3. REPORT TYPE AND DATES COVERED } \\
\text { Technical Memorandum }\end{array}$ \\
\hline
\end{tabular}

\section{ITLE AND SUBTITLE}

Description of the SSF PMAD DC Testbed Control System Data Acquisition Function

\section{Anastacio N. Baez, Michael Mackin, and Theodore Wright}

6. AUTHOR(S)

\section{PERFORMING ORGANIZATION NAME(S) AND ADDAESS(ES)}

National Aeronautics and Space Administration

Lewis Research Center

Cleveland, Ohio 44135-3191
WU $-474-42-10$

\section{FUNDING NuMBens}

8. Performing organization REPORT NUMBER

E-7286

10. SPONSOANGMONITORING AGENCY REPORT NUMBER

NASA TM-105843

Washington, D.C. 20546-0001

\section{SUPPLEMENTARY NOTES}

Prepared for the 27th Intersociety Energy Conversion Engineering Conference cosponsored by the ANS, SAE, ACS, AIAA, ASME, and IEEE, San Diego, California, August 3-7, 1992. Anastacio N. Baez, Michael Mackin, and Theodore Wright, NASA Lewis Research Center, Cleveland, Ohio. Responsible person, Anastacio N. Baez, (216) 433-5318.

12a. DISTRIBUTION/AVAILABILITY STATEMENT

12b. DISTAIBUTION CODE

Unclassified - Unlimited

Subject Category 62

13. ABSTRACT (Maximum 200 words)

The NASA Lewis Research Center in Cleveland, Ohio has completed the development and integration of a Power Management and Distribution (PMAD) DC Testhed. This lestbed is a reduced scale representation of the end to cnd, sources to loads, Space Station Frecdom Electrical Power System (SSF EPS). This unique facility is being used to demonstratc DC power generation and distribution, power management and control, and system operation techniques considered to be prime candidates for the Space Station Frecdom. A kcy capability of the testhed is its ability to be configured to address systcm level issucs in suppon of critical SSF program design milestoncs. Elcctrical power systcm control and operation issucs like source control, source regulation, system fault protection, end-to-end system stability, hcalth monitoring, resourcc allocation and resource management are being evaluated in the testhed. The SSF EPS control functional allocation between on-board computers and ground based systems is evolving. Initially, ground based systems will perform the bulk of power system control and operation. The EPS control system is required to continuously monitor and determine the current state of the power system. The DC Testbed Control System consists of standard controllers arranged in a hierarchical and distrihuted architecture. These controllers provide all the monitoring and conirol functions for the DC Testbed Electrical Power System. Higher Icvel controllers include the Power Managemeni Coniroller, Load Management Controlier, Operator Interface System, and a network of computer systems that perform some of the SSF Ground Based Control Centet Operation. The lower level controllers include Main Bus Switch Controllers and Photovoltaic Controllers. Power system status information is periodically provided to the higher level controllers to perform system control and operation. The data acquisition function of the control system is distributed among the various levels of the hierarchy. Data requirements are dictated by the control system ulgorithms being implemented at ewch level. This paper presents a functional description of the various levels of the testbed control system architecture and describes the data acquisition function and the status of its implementation.

\begin{tabular}{|c|c|c|c|}
\hline \multicolumn{3}{|c|}{$\begin{array}{l}\text { 14. SUBJECT TERMS } \\
\text { Computer systems; Space power systems; Control systems }\end{array}$} & \multirow{2}{*}{$\begin{array}{c}\text { 15. NUMBER OF PAGES } \\
8 \\
\text { 16. PAICE COOE } \\
\text { AO2 } \\
\end{array}$} \\
\hline & & & \\
\hline $\begin{array}{l}\text { 17. SECUATY CLASSIFICATION } \\
\text { OF REPOAT } \\
\text { Unclassified }\end{array}$ & $\begin{array}{l}\text { 18. SECUATY CLASSIFICATION } \\
\text { OF THIS PAGE } \\
\text { Unclassified }\end{array}$ & $\begin{array}{l}\text { 19. SECURTI CLASSIFICATION } \\
\text { OF ABSTRACT } \\
\text { Unclassified }\end{array}$ & 20. UMTTATION OF ABSTRACT \\
\hline JSN 7540-01-280-5500 & & & $\begin{array}{l}\text { indard Form } 298 \text { (Rev. } 2-89 \text { ) } \\
\text { scribed by ANSI Sid. Z39-18 }\end{array}$ \\
\hline
\end{tabular}


National Aeronautics and Space Administration

Lewis Research Center Cleveland, Ohio 44135

Otticial Business

Penalty for Private Use $\mathbf{5 3 0 0}$

FOURTH CLASS MAIL

ADDRESS CORRECTION REQUESTED

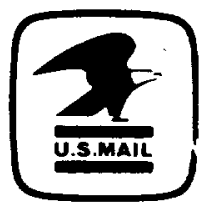

Postage and fees Pited

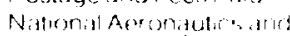

Sodce Aaministratim

NASAAST 
